\title{
Self-Configurable Current-Mirror Technique for Parallel RGB Light-Emitting Diodes (LEDs) Strings
}

\author{
Shaheer Shaida Durrani ${ }^{1}$ \\ Sustainable Energy and Power Electronics Research \\ (SuPER), Faculty of Electrical and Electronics Engineering \\ Technology, University Malaysia Pahang, Pekan, Malaysia
}

\author{
Asif Nawaz ${ }^{2}$ \\ ETS (Electronics) Faculty of Engineering \\ Higher College of Technology, Dubai, UAE \\ Muhamamd Shahzad ${ }^{3}$, Zeeshan Najam ${ }^{8}$ \\ Department of Electrical Engineering \\ MNS University of Engg and Tech, Multan, Pakistan \\ Rehan Ali Khan ${ }^{4}$ \\ Department of Electrical Engineering \\ University of Science and Technology, Bannu, Pakistan
}

\author{
Abu Zaharin Ahmad ${ }^{5}$ \\ Sustainable Energy and Power Electronics Research (SuPER) \\ Faculty of Electrical and Electronics Engineering Technology \\ University Malaysia Pahang, Pekan \\ Malaysia
}

Ahmed Ali Shah ${ }^{6}$

Department of Electrical Engineering Sukkur IBA University, Sukkur Pakistan

\author{
Sheeraz Ahmed ${ }^{7}$ \\ Department of Computer Science \\ Iqra National University, Peshawar \\ Pakistan
}

\begin{abstract}
Traditional current-mirror circuits require buck converter to deal with one fixed current load. This paper deals with improved self-adjustable current-mirror methods that can address different LED loads under different conditions with the help of one buck converter. The operating principle revolves around a dynamic and self-configurable combinational circuit of transistor and op-amp based current balancing circuit, along with their op-amp based dimming circuits. The proposed circuit guarantees uniformity in the outputs of the circuit. This scheme of current-balancing circuits omitted the need for separate power supply to control the load currents through different kinds of LEDs, i.e. RGB LEDs. The proposed methods are identical and modular, which can be scaled to any number of parallel current sources. The principle methodology has been successfully tested in Simulink environment to verify the current balancing of parallel LED strings.
\end{abstract}

Keywords-Current-balancing; LED driver; current mirror

\section{INTRODUCTION}

With the invention of visible phenomenon known as lightemitting diodes (LEDs), which is about a half-a-century old, they have now come into prominence for the past few years. Nowadays, LEDs are preferred choices since they are energy efficient and reliable [1]. The trend of utilizing LEDs is growing day by day and it is becoming the vital mandatory parts in some applications [2]. This has attracted the attention of many researchers, to study its applications. A lot of research has been going on to address various issued of LEDs, and one of the main issues is related to the imbalances of currents in parallel LED strings have drawn attention in recent years as LED technology is perceived as an emerging technology for replacing incandescent and fluorescent lamps. A most conventional method of current balancing is to connect each individual LED string [3] with resistor in series, which the power loss may lower the system efficiency. Various passive methods have been implemented with higher capacitors impedance to dominate the load impedance to achieve the current balancing [3] - [11]. In [12] - [18], implementing the combined methods to obtain the current balancing; however, the designed circuit contributed to the complicated ones.

In most approach, driving RGB LED arrays, need three converters, with their allied inductors. This causes the number of counts and increases the cost significantly [19]. As observed in [20], the RGB LED driver has been treated as a single input multi-output (SIMO), which is operated by a single inductor with different controllers for running RGB LED modules, to decrease the counts' numbers and to decrease the cost. SIMO drives RGB LEDs in a sequential scanning color scheme display (SCD) to reduce power consumption with fast response.

In [21], whenever the load varies suddenly, inductor current increases to get a new balance. Such the phenomenon is not considered suitable for the other converter of the system since the earlier converter takes time to get new balance and impact the output of other converters, which is in sequence. It eventually produces delay in the other converter's output and creates problems in getting uniform illumination of RGB LED modules. It is generally desired, to have a high degree of color stability for high-end applications like medical or museum lighting [22]. 
Meanwhile, [23] proposed the constant current sources for each load to energize the LEDs, i.e. a LED or string of LEDs. This method sounds easy, but it is costly and needs more components to the driver circuit, which creates the whole system complex. To address the issue, the current mirror (CM) is employed to remove imbalances in the currents of the strings. This solution requires very few components and is also less expensive solution in comparison to using only current sources. The CM driver has been recommended to be used for LED applications, where there is less likelihood of high value of current [24] and enhances the circuit response time and reduces complexity [25].

Dimming is an essential factor in the effective control of lighting and in saving energy, but it faces various challenges [26]. According to [21], while implementing analog dimming it is tough to maintain higher efficiency throughout the operation of wide range of diming. Contrary to this, PWM dimming approach has been used, and it is possible to utilize the full dimming range, since it has been observed that the color scheme of RGB LEDs can be controlled by changing the ratio of pulse width modulation (PWM) [27]. As stated in [20], there are three techniques available to control dimming phenomenon on system-level, and two techniques are available to control the dimming operation on string level. These methods need three different controllers as well as specific techniques like instant-duty-restoration (IDR), which makes the system costly and complicated.

Therefore, this article is meant to develop a CM circuit that facilitates different kinds of current controllers to be used efficiently. It also improves the time response of the entire system, under new desire output of dimming phenomenon. Secondly, to develop the RGB LED driver with single controller and inductor, which could overcome the issues related to the cross regulations. Thirdly, to build dimming circuit to exploit the full range with the technique of random PWM without using the method of IDR on string level as well as on the system level. This paper mainly discusses selfconfigurable CM methods for improving current imbalance in different parallel loads of color LEDs. The design method does not require a separate power supply for powering the CM circuit as well as their associate's circuits. Simulations have been conducted in the environment of Matlab/Simulink, to validate the proposed RGB LED driver.

\section{CIRCUIT DESCRIPTION}

The circuit configuration is for driving three string loads, in which each string connecting nine LEDs in parallel for red, green and blue LEDs. The loads have been designed to operate with buck converter, with the intent to provide PWM signals for the LED loads. The proposed particular LED driver system consists of a DC-DC buck converter or current generator, current mirror, LED loads and current regulator as depicted in Fig. 1.

The modified super diode in [28] for CM circuit is employed, while by applying some modifications i.e. diode of the super diode circuit with a resistor, along with the combination of op-amp based circuit for dimming with a properly biased transistor, to reduce power losses across transistor. The load arrays of RGB LEDs, along with their associates' circuits are shown in Fig. 2.

The proposed approach has a dynamic and selfconfigurable current-balancing circuit structure that allows the best current source (i.e., the smallest current source in the case of current balancing of parallel LED strings) to be selected. The proposed CM based current balancing circuit (refer Fig. 2) not requires 1) external power supply and 2) associated control circuit. Three parallel loads (LED strings, consisting of red, green, and blue LEDs) are connected to a selfconfigurable CM circuit. The transistors Q1 to Q3 (also called Q-transistors) represent the transistors used in a proposed CM circuit, such as the one shown in Fig. 2. Extra resistors that may be required for proper biasing of the circuit's transistors are not shown in Fig. 2 for the sake of simplicity.

Three transistor-based differential circuits are required along with super diode circuits for three difference loads connected in parallel as shown in Fig. 2. The differential circuit, which has the lowest load current, is selected as a current reference in a $\mathrm{CM}$ circuit to prevent the saturation of the transistors in the remaining differential circuits.

In analysis purposes, for the sake of simplicity, all the transistors are considered matched with the same current gain, $\beta$, and same resistive loads. If the current imbalances among the parallel current sources are not too significant (i.e., current inequality has been reduced), the transistor of the red LED with the smallest $V_{C E}$, drives the bases transistors of other differential circuits through current mirrors, forcing all the associated transistors with the super diode to work linearly.

Furthermore, the CM make changes in $V_{C E 3}$ and $V_{C E 2}$ to reduce $I_{1}$ and $I_{2}$ to follow the current reference $I_{l}$. This operating mode is based on the CM concept, except that there is a newness of a self-configurable feature that allows the best current source to be dynamically chosen as the reference current source for the CM operation.

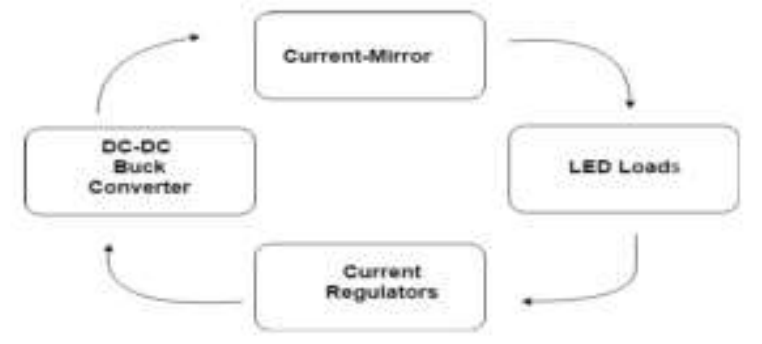

Fig. 1. Block Diagram of the Proposed LED Driver.

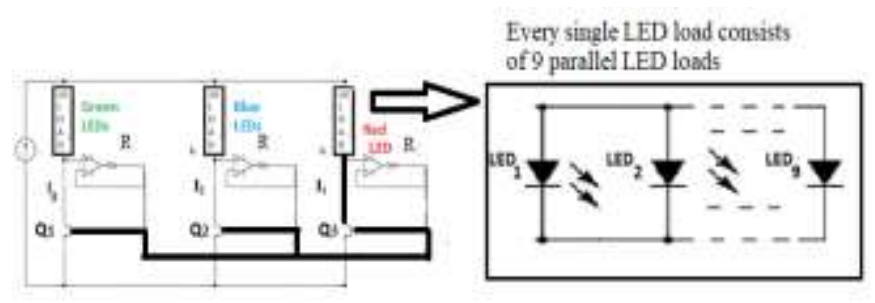

Fig. 2. Self-Configurable RGB LED Current Mirror Circuit. 
Using the assumption of $I_{1}>I_{2}>I_{3}$, and $V_{C E 3}>V_{C E 2}>$ $V_{C E I}$, the self-configurable principle can be illustrated with the aid of Fig. 2. The $V_{C E 3}$ being the smallest voltage across Q3 and the critical conducting path is highlighted with the bolded line in Fig. 2.

\section{DIMMING CONTROL MECHANISM-A HYBRID FuSION OF VolTAGE DIVIDER BIAS CIRCUIT AND COLLECTOR FEEDBACK RESISTOR}

A proper biasing is mandatory to operate transistor and to prevent it from going into saturation mode. It is a phenomenon related to the arrangement of dc collector current at a specific $\mathrm{dc}$ voltage by setting up an appropriate quiescent point. The biasing scheme is adopted by putting the base resistor $R_{B}$ in between the collector and the base terminals of the transistor as shown in Fig. 3. The resistor $R_{A}$ is connected between the emitter and the base terminals of the transistor, for sufficient biasing condition provided by the voltage drop across $R_{A}$. To lessen the losses, the kind of virtual resistance is proposed, which is not presented physically between the dimming circuit and the rest of the biasing circuit of the transistor. The dimming circuit is set to create different ground voltage references for the flow of load current from the biased transistor. Thus, by varying different voltage ground references, it becomes possible to create a kind of virtual resistance in the path of flow of load current, without having a real physical resistance over there.

All these LEDs need an individual dimming system. The applied dimming frequency to the dimming switching circuit is $4 \mathrm{Khz}$. The switching phenomenon has been implemented in each leg of the CM circuit, as shown in Fig. 4 and completed three string dimming is shown in Fig. 5.

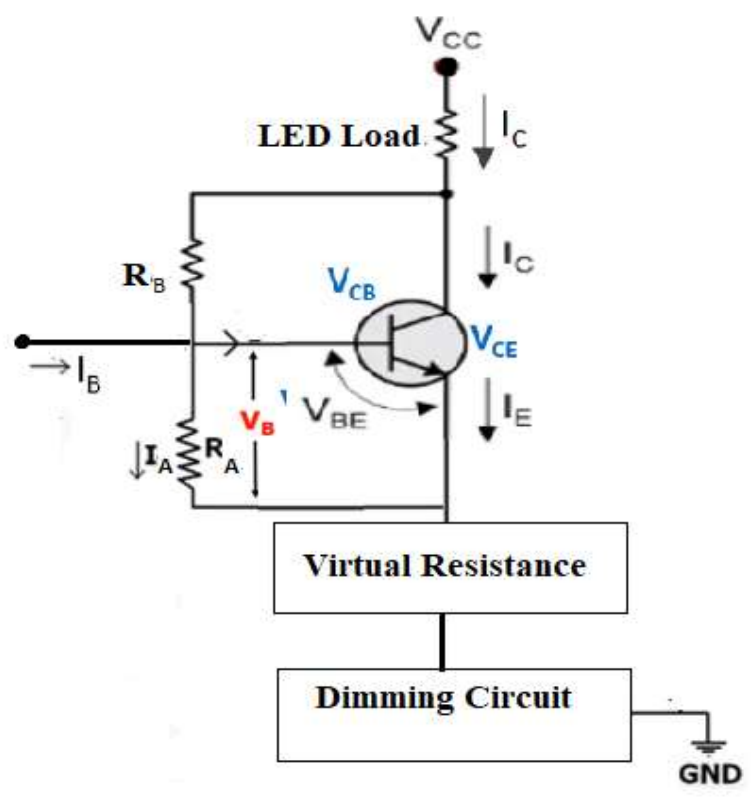

Fig. 3. Dimming Control Mechanism.

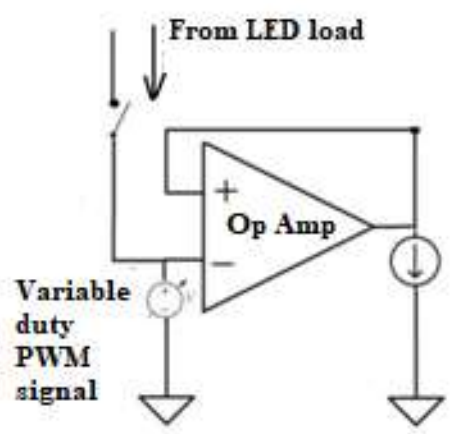

Fig. 4. Dimming/Switching Circuit and its Implementation in the Legs of CM.

\section{MATHEMATICAL ANANLYSIS FOR LED PARAMETER}

The total load consists of three loads i.e., the string of red LEDs, string of green LEDs and the string of blue LEDs. The parameters of RGB LEDs have been taken from [19]. The $R_{E Q U}$ represents the equivalent resistance of the red LED, with respect to its rated forward voltage and load current, whereas $R_{\text {OUT }}$ shows the parallel combination of the nine parallelconnected red LEDs in equation (2), same calculations have been done with respect to blue or green LEDs in equation (4). In the proposed scheme, there exists a total of three parallel strings, each individual string carries the load of parallel connected of nine LEDs with respect to their associated colors i.e. red, green and blue. The DC equivalent resistance $\left(R_{E Q U}\right)$ of the red LED has been found at their DC operating points [19] as follows:

$R_{E Q U}=\frac{V_{F W D}}{I_{L E D}}=\frac{1.9}{20 * 10^{-3}}=95 \Omega$

$R_{\text {OUT }}=R_{E Q U 1} / / \ldots \ldots \ldots . . / / R_{E Q U 9}=10.56 \Omega$

The currents forward voltages of the green and blue LEDs are similar. Their DC equivalent resistances of the green and blue LED can be found at their DC operating points as follows;

$R_{E Q U}=\frac{V_{F W D}}{I_{L E D}}=\frac{3.2}{20 * 10^{-3}}=160 \Omega$

$R_{\text {OUT }}=R_{E Q U 1} / / \ldots \ldots \ldots . . / / R_{E Q U 9}=17.78 \Omega$

Where, the $V_{F W D}$ denoted a forward voltage of LED, $I_{L E D}$ is the current pass through the LED string and $R_{\text {out }}$ is represented the total resistance of 9 LEDs in each string. It is noted that the red LED consumed less resistive than green and blue LEDs.

In the proposed dimming control circuit, it can introduce a new dimming level across a single and whole load array of LEDs. In principle, when dimming, the associated transistor of the string reduces the quantity of current through the load by raising the emitter's voltage, which eventually increases the collector's voltage of the transistor. It gives a freedom to LED running with a constant source of voltage with emitter coupled logic (ECL) topology, whereas avoiding transistors go to saturation. In which, the ECL is also known to be high speed integrated bipolar transistor logic. Hence, the combinational circuit of super diode, ECL topology and dimming control 
circuit provide a kind of controlling mechanism in providing a constant load current.

Refer to Fig. 5, $R_{e}$ is added in the designed as thermal runaway resistor, which has a small value to reduce the conduction losses and omitting the thermal runaway of transistors. The dimming on system level can be done easily while activating all the dimming circuits, which the $R_{l}$ is used to change the output voltage of op-amp of super diode to current. Meanwhile, the $R_{B}$ and $R_{A}$ are the feedback resistors and biasing resistors that used to reduce the power losses across the transistor. The efficiency $(\eta)$ of the LED driver can be computed by the ratio of the power dissipated by the LED to the total power dissipated in the string [29] as in following equation.

$\eta=\frac{P_{L E D}}{P_{L E D}+P_{\text {comp }}+P_{\text {tran }}} \times 100$

\section{A. Biasing Scheme}

In this approach, a proper biasing scheme is needed to do dimming uniformly throughout the whole system comprises of different resistances. The red LED has different resistive nature as compared to the green and blue LEDs, which leads to a differences of load current in red LED string. To make the system efficient, the load of red LED needs to be equal with other loads string, which can be made possible by adding external resistance (compensating resistance) in series with total red LEDs. However, such arrangements, contribute certain power losses as well as degrading the factor of efficiency $\eta$ as stated in equation (5).

To make the system comparatively efficient, a proper biasing scheme is needed. The biasing scheme adopted to all transistors $Q_{1}, Q_{2}$ and $Q_{3}$ using dual feedback transistor biasing scheme, as shown in Fig. 6. This sort of biasing method is beta $(\beta)$ dependent. The resistor $R_{B}$ is utilized for the collector to base feedback configuration to ensure transistor to always remain biased in the active region regardless of any value of the beta $(\beta)$ factor. The base bias voltage is dependent on the collector voltage, thus ensures good stability. $R_{A}$ has been added to the system to improve stability even more with respect to variations in beta $(\beta)$ by increasing the current flowing through the base biasing resistors. Thus, it increases the possibilities of a wide range of dimming spectrum.

A modification for biasing circuit of $Q_{3}$ transistor is carried out due to difference resistive load of red LED, by putting a base limiting resistance $\left(R_{b l}\right)$ to limit the base signal of $Q_{3}$ transistor (refer Fig. 6). The purpose of its introduction in the circuit is to improve the stability of the overall system by making $Q_{3}$ less responsive as compared to the response behavior of the other two $Q_{2}$ and $Q_{1}$ transistors by making beta $(\beta)$ of $Q_{3}$ less sensitive as compared to the transistors of other loads. Thus, the load current flowing through the $Q_{3}$, becomes almost identical to the load currents flowing through the transistors $Q_{2}$ and $Q_{1}$.

\section{VALIDATION}

A dimming approach for RGB LED driver has been set up to evaluate the performance of the self-configurable $\mathrm{CM}$ circuits. A DC-DC buck converter circuit has been set up to act as a current source for the proposed circuit.

\section{A. Self-Configurable Current-Mirror Circuit}

Since the red LED has less resistive value than other color LEDs, the modification of the dimming circuit is proposed so that the dimming can be conducted for all three consecutive string of RGB LEDs as shown in Fig. 5 and the designed parameters is depicted in Table I. The power of each string is always operated under the full load condition when the LED string is turned on, and no-load condition when LED string off. A SIMO driver for RGB LED with PWM dimming mechanism producing a relatively constant power efficiency at any dimming ratio, but in practice, there exists a small variation in their values as shown in the sets of calculations with references to Table II.

It has been observed that in a situation, where $R_{B L}$ is kept equal to $3 \Omega$, and alternately turning on and off of red, green and blue LEDs. It is found that a significant differences of load current red and blue/green LEDs as shown in Fig. 6 to Fig. 8. The efficiency of the red LED drop significantly and return increasing when rising the $R_{B L}$. Howewer, the load current increases accordingly as listed in Table II.

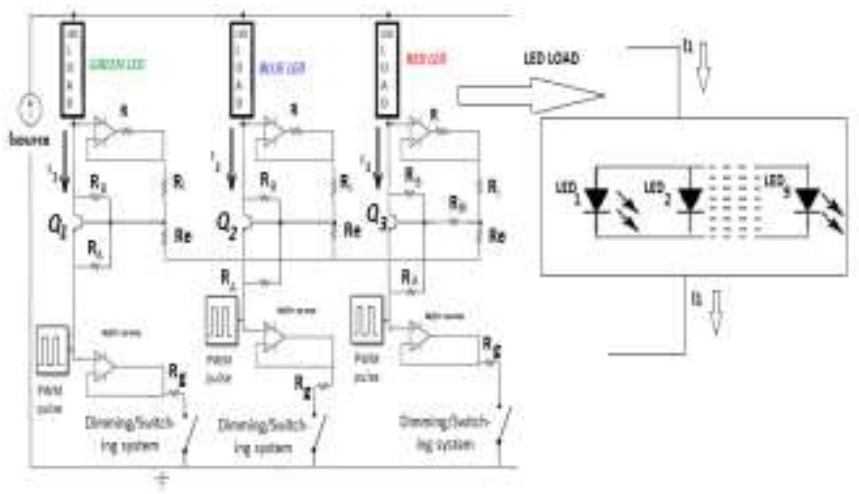

Fig. 5. Modified Self-Configurable RGB LED Current Mirror Circuit.

TABLE I. DESIGN SPECIFICATIONS OF AN OFF-LINE SIMO LED DRIVER

\begin{tabular}{|l|l|}
\hline Parameter & Value \\
\hline Input voltage (Vg) & 5 volts \\
\hline Maximum current & $22.17 \mathrm{~mA}$ \\
\hline Minimum current & $2.453 \mathrm{~mA}$ \\
\hline Main switching frequency & $300 \mathrm{Khz}$ \\
\hline Dimming frequency & $4 \mathrm{Khz}$ \\
\hline Inductor load current & $540 \mathrm{~mA}$ \\
\hline$R$ & $0.6 \Omega$ \\
\hline & $9 \Omega$ \\
\hline$R_{B}$ & $0.5 \Omega$ \\
\hline$R_{A}$ & $2 \Omega$ \\
\hline$R_{e}$ & $3 \Omega$ \\
\hline$R_{B L}$ & $20 \Omega$ \\
\hline$R_{l}$ & $1 \Omega$ \\
\hline$R_{g}$ & \\
\hline &
\end{tabular}


TABLE II. DESIGN SPECIFICATIONS OF AN OFF-LINE SIMO LED DRIVER

\begin{tabular}{|l|l|l|l|l|l|l|}
\hline & $\boldsymbol{R}_{\boldsymbol{B} \boldsymbol{L}}$ & $\begin{array}{l}\text { Load } \\
\text { Current } \\
\text { through } \\
\text { Red } \\
\text { LED }\end{array}$ & $\begin{array}{l}\text { Load } \\
\text { Current } \\
\text { through } \\
\text { Green/Blue } \\
\text { LED }\end{array}$ & $\begin{array}{l}\Delta \\
\text { Current }\end{array}$ & $\begin{array}{l}\text { Red } \\
\text { LED } \\
\eta\end{array}$ & $\begin{array}{l}\text { Blue/Green } \\
\text { LED } \eta\end{array}$ \\
\hline 1. & $3 \Omega$ & $210 \mathrm{~mA}$ & $220.4 \mathrm{~mA}$ & 10.4 & $21 \%$ & $57 \%$ \\
\hline 2. & $50 \Omega$ & $\begin{array}{l}221.5 \\
\mathrm{~mA}\end{array}$ & $211.5 \mathrm{~mA}$ & 10 & $45 \%$ & $51 \%$ \\
\hline 3. & $100 \Omega$ & $\begin{array}{l}222.4 \\
\mathrm{~mA}\end{array}$ & $210.8 \mathrm{~mA}$ & 11.6 & $48 \%$ & $51 \%$ \\
\hline 4. & $200 \Omega$ & $\begin{array}{l}222.9 \\
\mathrm{~mA}\end{array}$ & $210.5 \mathrm{~mA}$ & 12.4 & $52 \%$ & $51 \%$ \\
\hline 5. & $500 \Omega$ & $\begin{array}{l}223.2 \\
\mathrm{~mA}\end{array}$ & $210.5 \mathrm{~mA}$ & 12.7 & $52 \%$ & $51 \%$ \\
\hline 6. & $1000 \Omega$ & $\begin{array}{l}223.5 \\
\mathrm{~mA}\end{array}$ & $210.5 \mathrm{~mA}$ & 13 & $52 \%$ & $51 \%$ \\
\hline
\end{tabular}

\section{DIMMING}

There are two options of dimming available for the LEDs, based on the operations of the dimming circuits, associated with their LED loads. First is associated with the dimming available for every individual LED load, which is by controlling their associated dimming circuits. Second option is to facilitate the user to do dimming operations simultaneously for all string. The proposed LED driver has a $5 \mathrm{~V}$ DC voltage from buck DC-DC converter. At first, the validation is carried out through the circuit simulation.

\section{A. String-Level Dimming Evaluation}

In this dimming mode, the technique of employing super diode along with its dimming circuit is purposed to evaluate the circuit ability for controlling three separate independent different load currents. The load currents of $I_{1}, I_{2}, I_{3}$ and dimming gate signal are shown in Fig. 6. The dimming signal is set from $10 \%$ to $90 \%$ of range. Fig. 6 to Fig. 8 show the current waveforms $\left(I_{1}, I_{2}\right.$ and $\left.I_{3}\right)$, as well as the corresponding gate signals for the dimming circuits, associated with their load currents. The reference output currents for the three LED strings are respectively set at $210 \mathrm{~mA}, 220 \mathrm{~mA}$ and $220 \mathrm{~mA}$, and the dimming duty cycles is set at $10 \%$. For further analysis, the $10 \%$ dimming signal is equal to on-state while $90 \%$ dimming signal is off-state.

The result showed that during the on-state, the output currents are regulated to the reference. In comparison, the three LED strings are set identical of dimming parameters, i.e., the same on-state current references and dimming ratios frequency. The dimming operation is generated at differences timing for showing the robustness of the system. These waveforms are almost identical to the voltage drops across their associated loads. The behaviors waveforms show that the loads are operating according to their power ratings.

In Fig. 6, illustrating the red LED behavior of load current, voltage across the load and dimming signal, respectively. It is clearly visible that the voltage across the red LEDs following the load current. Referring to dimming signal, the on and off states are implemented. For $10 \%$ of dimming, the maximum amount of current is found approximately to $210 \mathrm{~mA}$, while during $90 \%$ of dimming, the minimum amount of current is found drop to around $25 \mathrm{~mA}$. The loading effects could be seen during off-state for the individual LEDs, which is not more than $2 \%$ of the full load current. For others string, the green and blue LEDs are shown in Fig. 7 and 8, respectively. It is clearly seen that the signal behavior comparable with red LED load. During 10\% dimming, the current flow comparable to the reference of $220 \mathrm{~mA}$ while during off-state drop to around $25 \mathrm{~mA}$ and $30 \mathrm{~mA}$ for green and blue respectively. The loading effects also could be seen at off-state period.

On the other hand, through the proposed dimming mechanism, the buck dc-dc converter does not require closedloop control to control the dimming mechanism as shown in Fig. 9. As a result, the range of the dimming signals could be extended from $10 \%$ up to $90 \%$ dimming, which is almost to the full range dimming.

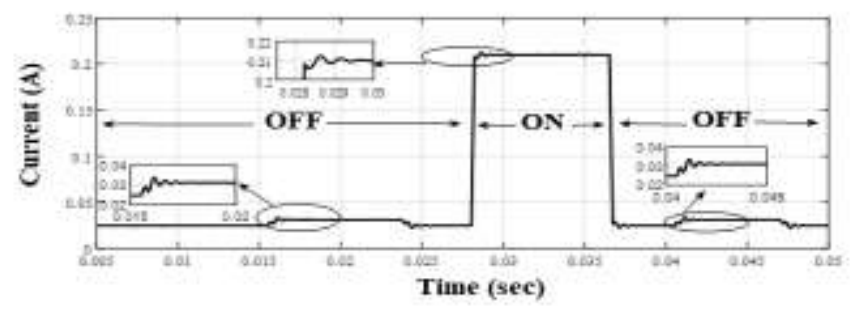

(a)

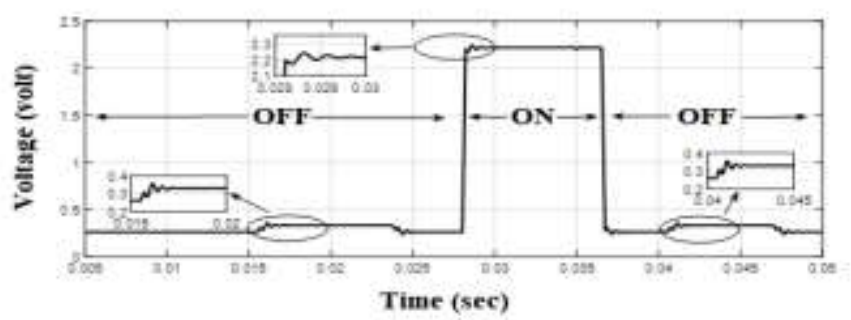

(b)

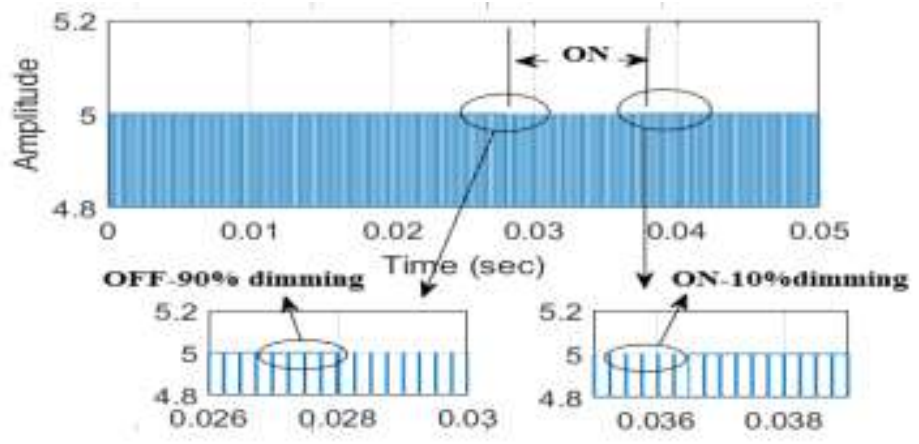

(c)

Fig. 6. Waveforms of the Output with PWM Dimming of $10 \%$ and $90 \%$ (a) Load Current Response of Red LEDs (b) Voltage Across the Red LEDs (c) Close-up view of the widths of the PWM Signals for Turning off and on the red LEDs. 


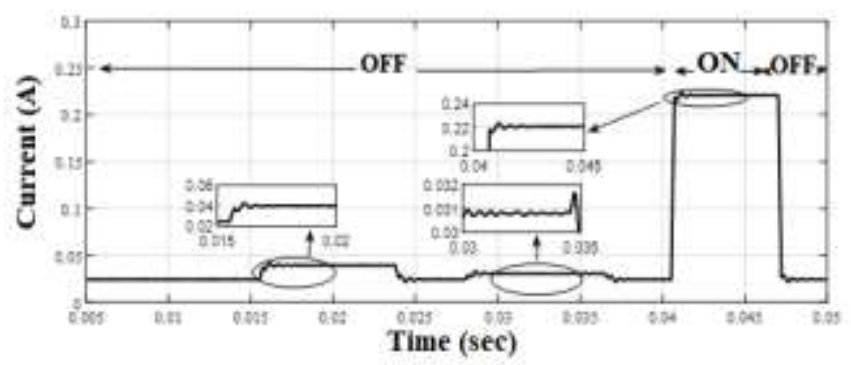

(a)

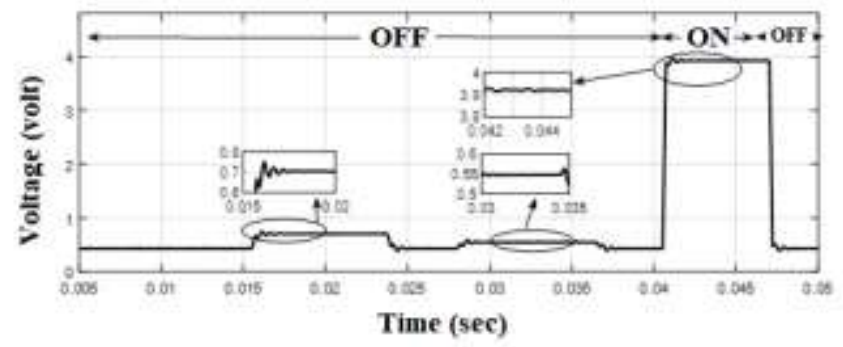

(b)

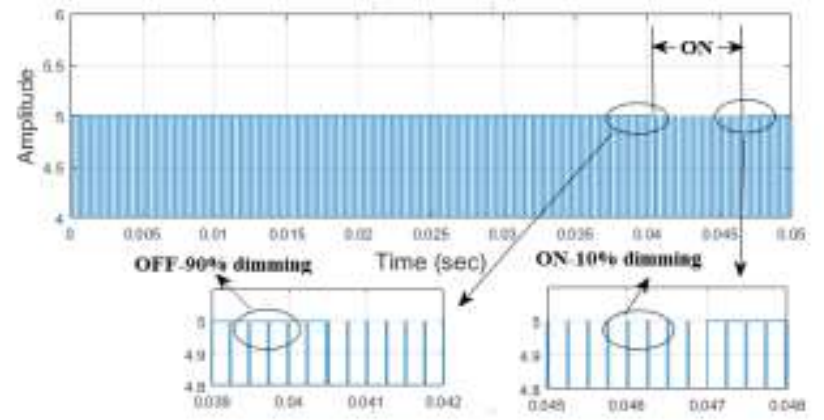

(c)

Fig. 7. Waveforms of the Output with PWM Dimming of $10 \%$ and $90 \%$ (a) Load Current Response of Green LEDs (b) Voltage across the Green LEDs (c) Close-up view of the widths of the PWM Signals For turning off and on the Green LEDs.

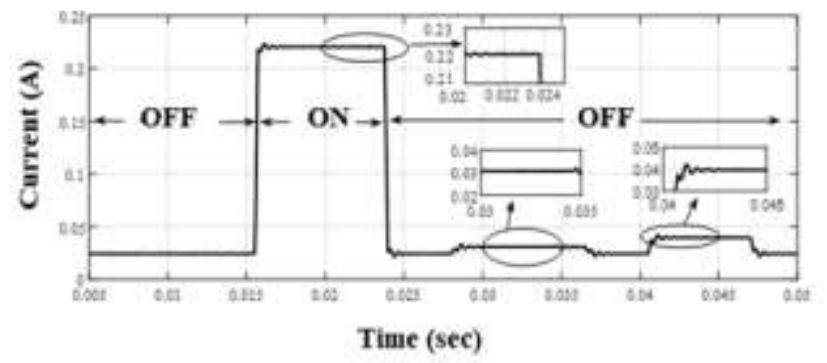

(a)

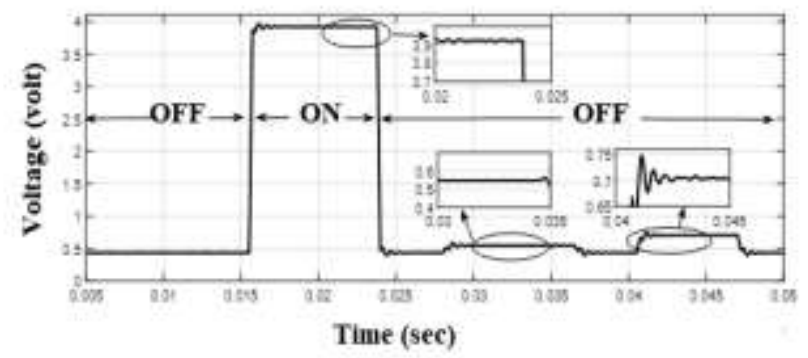

(b)

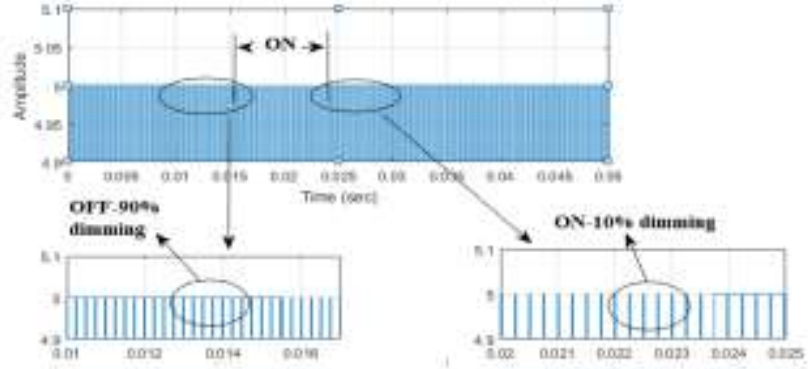

(c)

Fig. 8. Waveforms of the Output with PWM Dimming of $10 \%$ and $90 \%$ (a) Load Current Response of Blue LEDs (b) Voltage Across the Blue LEDs (c) Close-up view of the widths of the PWM Signals for Turning off and on the Blue LEDs.

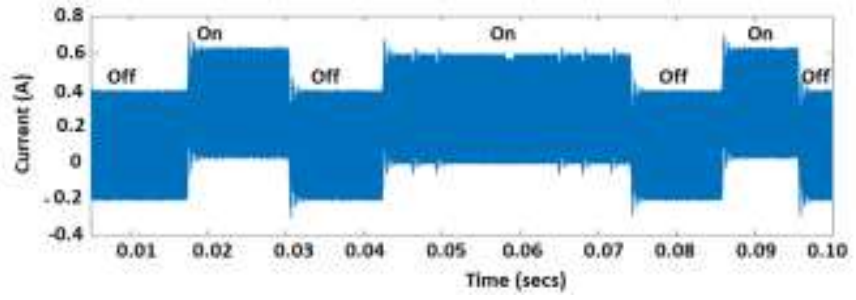

Fig. 9. Inductor's Load Current Variations Corresponding to the Turning off and on of the LEDs through Dimming Circuits.

\section{B. System Level Dimming Evaluation}

Second scenario, the combinational circuits of super diode and dimming circuit for all the load strings are analyzed. Simultaneously operating in the range of $10 \%$ to $90 \%$ of dimming signal is implemented. In Fig. 10, showing the results for load current, voltage across the load and dimming signal respectively. The behavior is comparable according to the on and off states conditions. Nevertheless, in the system level dimming, all the loads take current spontaneously and share their currents with other loads. Since the current at green and blue LEDs are slightly more than red LEDs, hence the surplus current goes to the red LEDs due to the proposed circuit act to balance current through the string with differences loads. As the result, the load current at red LEDs increases significantly. It is showed the effectiveness of the proposed mirroring circuit in managing the current sharing. It is different with the string level dimming, which is the green and blue LEDs are not activated when the red LEDs on. The process same with other LEDs string turning on. Thus, avoiding the phenomenon of current balancing from other string LEDs.

In Tables III and IV, listing the computed power losses through the LED string loads (i.e.; red, green and blue LEDs). The condition is activating the dimming simultaneously for three string LEDs loads to figure out the efficiency. Through the measurement, the maximum load currents for red and green/blue LEDs are $221.8 \mathrm{~mA}$ and $219.0 \mathrm{~mA}$ respectively. For red LEDs, the measured voltage drop across the transistor and current flow through the transistor are $2.111 \mathrm{~V}$ and 229.6 $\mathrm{mA}$, respectively. Meanwhile, $0.56 \mathrm{~V}$ and $243.7 \mathrm{~mA}$ are measured through green/blue LEDs. Hence, the power losses through transistor for red and green/blue LEDs could be computed accordingly as shown in Tables III and IV. 


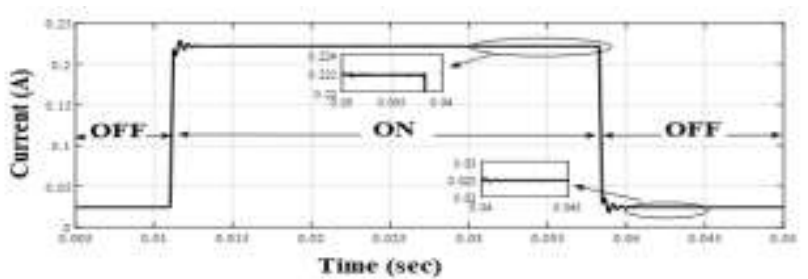

(a)

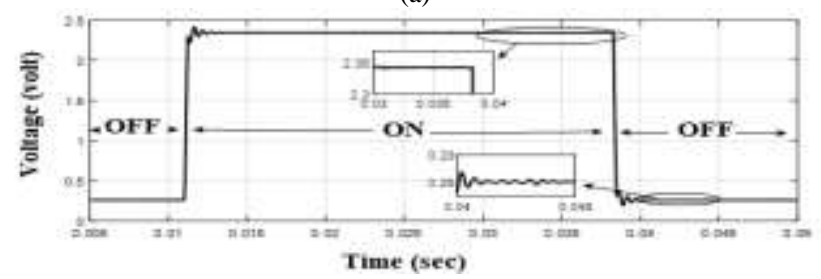

(b)

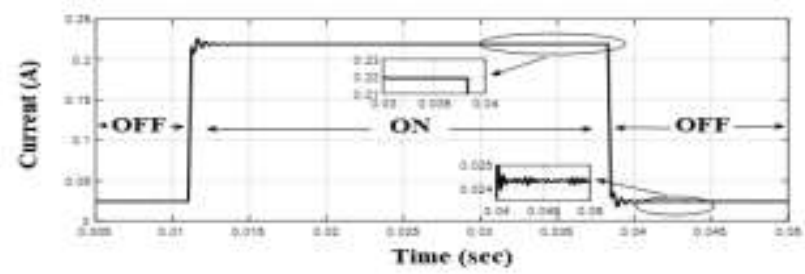

(c)

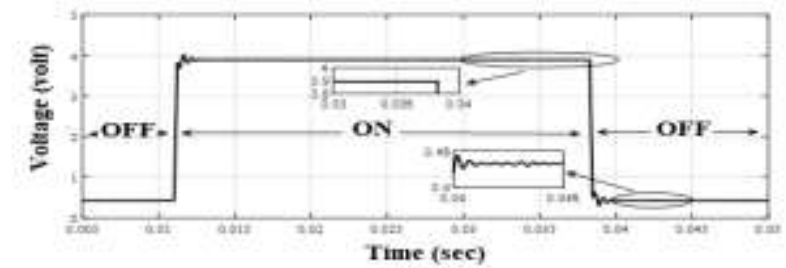

(d)

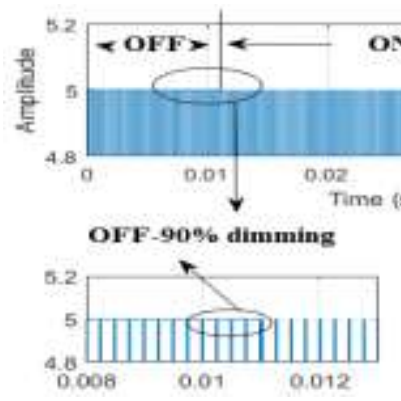

(e)

Fig. 10. Waveforms of the System Output with PWM Dimming of $10 \%$ and 90\% (a) Load Current Response of Red LEDs (b) Voltage Across the Red LEDs (c) Load Current Response of Green/Blue LEDs (d) Voltage Across the Green/Blue LEDs (e) Close-up view of the widths of the PWM Signals for Turning off and on the LEDs.

Thus, the computation efficiency for red $\left(\eta_{r_{-} \max }\right)$ LEDs is $52 \%$, and $86 \%$ efficiency $\left(\eta_{g b_{-} \max }\right)$ for green/blue LEDs.

On the other hand, the measurement of power losses at minimum load currents for red and green/blue LEDs are 24.58 $\mathrm{mA}$ and $24.27 \mathrm{~mA}$. In red LEDs string, the measured transistor voltage drops and current pass through the transistor are $0.234 \mathrm{~V}$ and $25.44 \mathrm{~mA}$ respectively. Meanwhile, for green/blue LEDs string, $62.04 \mathrm{mV}$ and $27.0 \mathrm{~mA}$ are measured for voltage drop and current through the transistor. Thus, the power losses can be estimated as listed in Tables V and VI.
TABLE III. POWER LOSSES FOR RED LEDS AT MAXIMUM CURRENT

\begin{tabular}{|l|l|}
\hline Red LEDs $\left(P_{L E D}\right)$ & $520 \mathrm{~mW}$ \\
\hline Transistor terminal $\left(P_{\text {trans }}\right)$ & $480 \mathrm{~mW}$ \\
\hline
\end{tabular}

TABLE IV. POWER LOSSES FOR GREEN/BLUE LEDS AT MAXIMUM CURRENT

\begin{tabular}{|l|l|}
\hline Green/Blue LEDs $\left(P_{\text {LED }}\right)$ & $853 \mathrm{~mW}$ \\
\hline Transistor terminal $\left(P_{\text {trans }}\right)$ & $136.3 \mathrm{~mW}$ \\
\hline
\end{tabular}

TABLE V. POWER LOSSES FOR RED LEDS AT MiNIMUM CURRENT

\begin{tabular}{|l|l|}
\hline Red LEDs $\left(P_{L E D}\right)$ & $6.4 \mathrm{~mW}$ \\
\hline Transistor terminal $\left(P_{\text {trans }}\right)$ & $6 \mathrm{~mW}$ \\
\hline
\end{tabular}

TABLE VI. POWER LOSSES FOR GREEN/BLUe LEDS AT MINIMUM CURRENT

\begin{tabular}{|l|l|}
\hline Green/Blue LEDs $\left(P_{L E D}\right)$ & $10.5 \mathrm{~mW}$ \\
\hline Transistor terminal $\left(P_{\text {trans }}\right)$ & $2 \mathrm{~mW}$ \\
\hline
\end{tabular}

Therefore, the estimation efficiency at minimum load current for red $\left(\eta_{r_{-} \min }\right)$ LEDs is $52 \%$, and for green/blue $\left(\eta_{g b \_\min }\right)$ LEDs is $85 \%$.

\section{VII.CONCLUSION}

In this paper, an investigation has been carried out to determine the effectiveness of the current mirroring circuit to regulate the different LED loads, while keeping and regulating the minimum power losses through the string module. Though green and blue LEDs have same resistive in nature, which is slightly difference with red LED, the current mirror has successfully managed to bring identical currents passing through the loads. The proposed circuit could be implementing two option of dimming, which are individual string level and system level dimming. It is show flexibility of the dimming purposes. Another advantage, it has found that, the analyses has done the measurement of precisely dimming from $10 \%$ to $90 \%$ of dimming range. It is showed the effectiveness of the proposed LED driver and dimming circuit for color LED string.

\section{REFERENCES}

[1] K.H. LiW.Y. FuH.W. Cho,: Chip-scale GaN integration, Progress in quantum electronics 70 (2020) 100247.

[2] Durrani S.S., Zaharin A., Hassan B., Ishak R.B. (2020) Comparative Analysis for LED Driver with Analog and Digital Controllers. In: Kasruddin Nasir A. et al. (eds) InECCE2019. Lecture Notes in Electrical Engineering, Vol 632. Springer, Singapore. https://doi.org/10.1007/978981-15-2317-5_73.

[3] Mudassar Khatib.: Ballast Resistor Calculation-Current Matching in Parallel LEDs, Texas Instruments, April 2009.

[4] W. K. Lun, ., K. H. Loo, ., S. C. Tan, ., Y. M. Lai, ., C. K. Tse,:Bi level Current Driving Technique for LEDs, IEEE Trans. Power Electronics, vol. 24, no. 12, pp. 2920-2930, 2009.

[5] C. H. Lin, ., T. Y. Hung, C. M. Wang, ., d K. J. Pai,:A Balancing Strategy and Implementation of Current Equalizer for High Power LED Backlighting, IEEE Power Electronics and Drive Systems PEDS '07. 7th International Conference, pp. 1613-1617, Nov. 2007.

[6] K. H. Jung, J. W. Yoo, ., C. Y. Park,:A Design of Current Balancing Circuit for Parallel Connected LED strings using Balancing Transformers, IEEE Power Electronics and ECCE Asia (ICPE \& ECCE), pp. 528-535, 2011. 
[7] K. I. Hwu., S. C. Chou: A Simple Current-Balancing Converter for LED Lighting" IEEE Applied Power Electronics Conference (APEC) Proc, pp. 587-590, February 2009.

[8] Y. Hu, M. M. Jovanovic: A New Current-Balancing Method for Paralleled LED Strings, IEEE Applied Power Electronics Conference (APEC), 26th Annual, Mar. 2011.

[9] J. Zhang, ., J. Wang, . X. Wu,:A Capacitor-Isolated LED Driver with Inherent Current Balance Capability, IEEE Trans. Industrial Electronics, vol. 59, no. 4, pp. 1708-1716, 2011.

[10] S. Choi, P. Agarwal, ., T. Kim, ., J. Yang, ., ., B. Han, Symmetric Current Balancing Circuit for Multiple DC loads, IEEE Applied Power Electronics Conference (APEC) Proc., pp. 512-518, 2010.

[11] S. M. Baddela, D. S. Zinger, "Parallel Connected LEDs Operated at High Frequency to Improve Current Sharing," IEEE Industry Applications Conference, 2004.

[12] J.Wang, J. Zhang, Y. Shi., Z. Qian: A Novel High Efficiency and LowCost Current Balancing Method for Multi-LED Driver, IEEE Energy Conversion Congress and Exposition (ECCE), pp. 2296-2301, 2011.

[13] S. H. Cho, S. H. Lee, ., S. S. Hong, D. S. Oh . S.K. Han, "HighAccuracy and Cost-Effective Current-Balanced Multichannel LED Backlight Driver Using Single-Transformer, IEEE Power Electronics and ECCE Asia 8th International Conference, pp. 520-527, 2011.

[14] S. Zhang, Q. Chen, J. Sun, M. Xu, Y. Qiu, High-Accuracy Passive Current Balancing Schemes for Large-Scale LED Backlight System, IEEE Applied Power Electronics Conference and Exposition (APEC), pp. 723-727, 2011.

[15] J. Wamg, J. Zhang, ., X. Huang, . L. Xu,:A Family of Capacitive Current Balancing Methods for Multi-Output LED Drivers, IEEE Applied Power Electronics Conference and Exposition (APEC), pp. 2010-2046, 2011."A Dimmable Light-Emitting Diode ".

[16] W. Chen, S. Y. R. Hui,:A Dimmable Light-Emitting Diode (LED) Driver with cascaded mag-amp postregulators for multistring applications, IECON Conference on IEEE Industrial Electronics Society, pp. 2523-2528, 2010.

[17] T. Werner, J. Pforr,: A Novel Low-Cost Current-Sharing Method for Automotive LED Lighting System, Power Electronics and Applications European Conference, pp. 1-10, 2009.

C. Zhao, X.Xie, ., S. Liu,:A Precise Passive Current Balancing Method for Multi-Output LED Drivers, IEEE Trans. Power Electronics, 2011.

[18] J. Hasan, S. S. Ang,:A high-efficiency digitally controlled RGB driver for LED pixels, IEEE Trans. Ind. Appl., vol. 47, no. 6, pp. 2422-2429, Nov./Dec. 2011.
[19] S. Li, Y. Guo, A. Lee, S. Tan, ., S. Y. Hui,:An off-line single-inductor multiple-outputs LED driver with high dimming precision and full dimming range, IEEE Trans. Power Electron., vol. PP, no. 99, pp. 1-1, 2017.

[20] Patra, P., Patra, A., Misra, N.: A single-inductor multiple-output switcher with simultaneous buck, boost, and inverted outputs, IEEE Trans. Power Electron., 2012, 27, (4), pp. 1936-1951

[21] Kwai Hei Li, Member, IEEE, Yuk Fai Cheung, Weijian Jin, Wai Yuen Fu, Albert Ting Leung Lee, Siew Chong Tan, Shu Yuen Hui, and Hoi Wai Choi ,:" InGaN RGB Light-Emitting Diodes With Monolithically Integrated Photodetectors for Stabilizing Color Chromaticity". IEEE Transactions on Industrial Electronics, vol. 67, no. 6, June 2020.

[22] Muhinthan Murugesu, Osram Opto Semiconductors Vector, Current distribution in parallel LED strings, Vector - journal of the Institution of Certificated Mechanical and Electrical Engineers (ICMEESA), and the Illumination Engineering Society of South Africa (IESSA), January 2013.

[23] H.-J. Chiu, ., S.-J. Cheng,: LED backlight driving system for largescale LCD panels, IEEE Trans. Industrial Electronics, Vol. 54, No. 5, pp. 2751-2760, Oct. 2007.

[24] Shaheer Shaida Durrani, abu Zaharin ahmed,: An Efficient Digitally Controlled for RGB LED Driver, IEEE ICETAS Conference Bahrain, 2017.

[25] Vili Väinölä, ., Sina khamehchi ,., Hassan rouhi, ., Tapio kukkonen, ., Visnukumar Murugesan,: Project \#21 Illumination and colour control in flicker-free LED lighting, Aalto University, School of Electrical Engineering Automation and Electrical Engineering (AEE) Master's Programme ELEC-E8002 \& ELEC-E8003 Project work course Year 2017.

[26] Xin Yu Guo, Guo Chun Wan, and Mei Song Tong: An Intelligent Control System of Music Rhythms by RGB-LED Lamp. 2019 PhotonIcs \& Electromagnetics Research Symposium | Fall (PIERS | FALL), Xiamen, China, $17\{20$ December.

[27] Sung-Jin Choi, : Adaptive Current-Mirror LED Driver employing Superdiode Configuration, IEEE International Conference on Industrial Technology.2014.

[28] J. Falin. (2008, 4Q). Compensating and measuring the control loop of a high-power LED driver. Analog Appl.J. [Online]. pp. 14-17. Available: http://www.ti.com/lit/an/slyt308/slyt308.pdf. 